\title{
ADAPTIVE CONTROL AND SYNCHRONIZATION OF THE CAI SYSTEM
}

\author{
Sundarapandian Vaidyanathan ${ }^{1}$ \\ ${ }^{1}$ Research and Development Centre, Vel Tech Dr. RR \& Dr. SR Technical University \\ Avadi, Chennai-600 062, Tamil Nadu, INDIA \\ sundarvtu@gmail. com
}

\begin{abstract}
This paper derives new results for the adaptive control and synchronization design of the Cai system (2007), when the system parameters are unknown. Cai system is one of the paradigms of 3-D chaotic systems discovered by Cai and Tan (2007). In this paper, we first construct an adaptive controller to stabilize the Cai system to its unstable equilibrium at the origin. Then we build an adaptive synchronizer to achieve global chaos synchronization of the identical Cai systems with unknown parameters. The results derived for adaptive stabilization and adaptive synchronization for the Cai systems have been established using adaptive control theory and Lyapunov stability theory. Numerical simulations have been shown to demonstrate the effectiveness of the adaptive control and synchronization schemes derived in this paper for the Cai system.
\end{abstract}

\section{KEYWORDS}

Adaptive Control, Chaos, Chaotic Systems, Synchronization, Cai System.

\section{INTRODUCTION}

Nonlinear dynamical systems, which are extremely sensitive to changes in initial conditions, are known as chaotic systems. Chaotic systems exhibit random-like behaviour in its deterministic motion. Experimentally, chaos was first discovered by Lorenz ([1], 1963), while he was simulating weather models. A chaotic system simpler than the Lorenz system was proposed by Rössler ([2], 1976).

The control of chaotic systems is to design state feedback control laws that stabilizes the chaotic systems around the unstable equilibrium points. Active control technique is used when the system parameters are known and adaptive control technique is used when the system parameters are unknown [3-4].

Synchronization of chaotic systems is a phenomenon that may occur when two or more chaotic attractors are coupled or when a hyperchaotic attractor drives another hyperchaotic attractor. In the last two decades, there has been significant interest in the literature on the synchronization of chaotic and hyperchaotic systems [5-16].

In 1990, Pecora and Carroll [5] introduced a method to synchronize two identical chaotic systems and showed that it was possible for some chaotic systems to be completely synchronized. From then on, chaos synchronization has been widely explored in a variety of fields including physical systems [6], chemical systems [7], ecological systems [8], secure communications [9-10], etc.

DOI : 10.5121/ijci.2012.1302 
The pioneering work by Pecora and Carroll (1990) has been followed by a variety of impressive approaches in the literature such as the sampled-data feedback method [11], OGY method [12], time-delay feedback method [13], backstepping method [14], active control method [15-20], adaptive control method [21-25], sliding mode control method [26-28], etc.

This paper is organized as follows. In Section 2, we give a description of the Cai chaotic system (Sprott, [29], 1994). In Section 3, we derive results for the adaptive control of Cai chaotic system with unknown parameters. In Section 4, we derive results for the adaptive synchronization of the identical Cai chaotic systems with unknown parameters. Section 5 contains a summary of the main results derived in this paper.

\section{SYSTEM DESCRIPTION}

The Cai system ([29], 2007) is described by the 3D dynamics

$$
\begin{aligned}
& \dot{x}_{1}=a\left(x_{2}-x_{1}\right) \\
& \dot{x}_{2}=b x_{1}+c x_{2}-x_{1} x_{3} \\
& \dot{x}_{3}=x_{1}^{2}-d x_{3}
\end{aligned}
$$

where $x_{1}, x_{2}, x_{3}$ are the state variables of the system and $a, b, c, d$ are constant, positive parameters of the system.

The system (1) is chaotic when the parameter values are taken as

$$
a=20, b=14, c=10.6 \text { and } d=2.8
$$

Figure 1 describes the strange attractor of the Cai system (1).

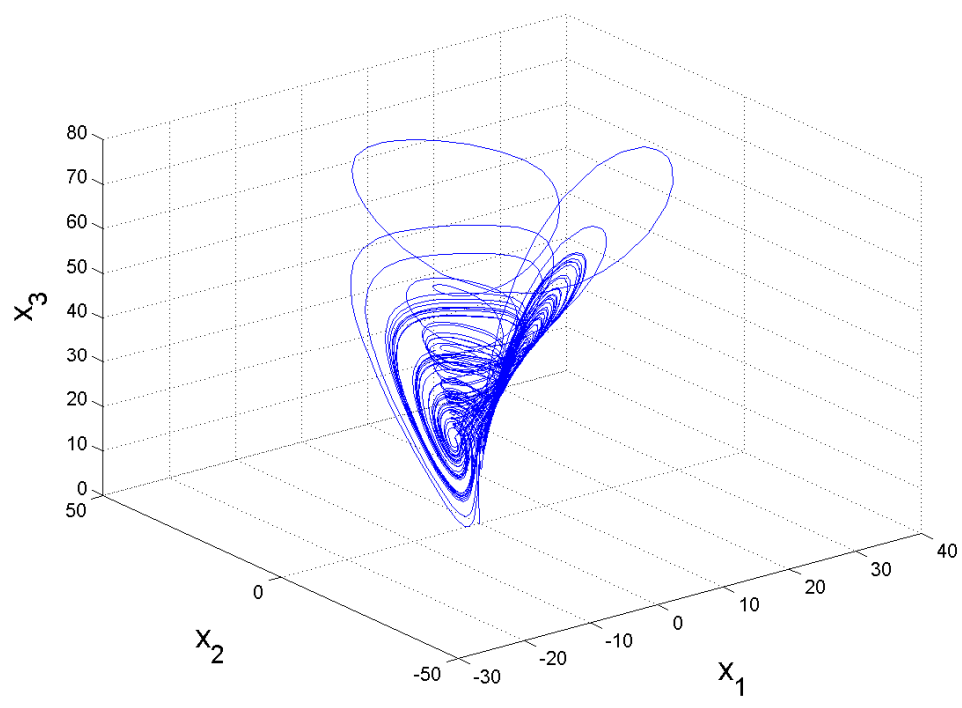

Figure 1. The Strange Attractor of the Cai System 
When the parameter values are taken as in (2) for the Cai chaotic system (1), the system linearization matrix at the equilibrium point $E_{0}=(0,0,0)$ is given by

$$
A=\left[\begin{array}{ccc}
-20 & 20 & 0 \\
14 & 10.6 & 0 \\
0 & 0 & -2.8
\end{array}\right]
$$

which has the eigenvalues

$$
\lambda_{1}=-27.3736, \lambda_{2}=-2.8 \text { and } \lambda_{3}=17.9736
$$

Since $\lambda_{3}$ is an unstable eigenvalue of $A$, it follows from Lyapunov stability theory [30] that the Cai system (1) is unstable at the equilibrium point $E_{0}=(0,0,0)$.

\section{Adaptive Control of the Cai Chaotic System}

\subsection{Theoretical Results}

In this section, we design adaptive control law for globally stabilizing the Cai system (2007), when the parameter values are unknown.

Thus, we consider the controlled Cai system, which is described by the 3D dynamics

$$
\begin{aligned}
& \dot{x}_{1}=a\left(x_{2}-x_{1}\right)+u_{1} \\
& \dot{x}_{2}=b x_{1}+c x_{2}-x_{1} x_{3}+u_{2} \\
& \dot{x}_{3}=x_{1}^{2}-d x_{3}+u_{3}
\end{aligned}
$$

where $u_{1}, u_{2}$ and $u_{3}$ are feedback controllers to be designed using the states $x_{1}, x_{2}, x_{3}$ and estimates $\hat{a}, \hat{b}, \hat{c}, \hat{d}$ of the unknown parameters $a, b, c, d$ of the system.

In order to ensure that the controlled system (3) globally converges to the origin asymptotically, we consider the following adaptive control functions

$$
\begin{aligned}
& u_{1}=-\hat{a}\left(x_{2}-x_{1}\right)-k_{1} x_{1} \\
& u_{2}=-\hat{b} x_{1}-\hat{c} x_{2}+x_{1} x_{3}-k_{2} x_{2} \\
& u_{3}=-x_{1}^{2}+\hat{d} x_{3}-k_{3} x_{3}
\end{aligned}
$$

where $\hat{a}, \hat{b}, \hat{c}$ and $\hat{d}$ are estimates of the parameters $a, b, c$ and $d$, respectively, and $k_{i},(i=1,2,3)$ are positive constants.

Substituting the control law (4) into the controlled Cai dynamics (3), we obtain 


$$
\begin{aligned}
& \dot{x}_{1}=(a-\hat{a})\left(x_{2}-x_{1}\right)-k_{1} x_{1} \\
& \dot{x}_{2}=(b-\hat{b}) x_{1}+(c-\hat{c}) x_{2}-k_{2} x_{2} \\
& \dot{x}_{3}=-(d-\hat{d}) x_{3}-k_{3} x_{3}
\end{aligned}
$$

Let us now define the parameter errors as

$$
e_{a}=a-\hat{a}, \quad e_{b}=b-\hat{b}, \quad e_{c}=c-\hat{c} \text { and } e_{d}=d-\hat{d}
$$

Using (6), the closed-loop dynamics (5) can be written compactly as

$$
\begin{aligned}
& \dot{x}_{1}=e_{a}\left(x_{2}-x_{1}\right)-k_{1} x_{1} \\
& \dot{x}_{2}=e_{b} x_{1}+e_{c} x_{2}-k_{2} x_{2} \\
& \dot{x}_{3}=-e_{d} x_{3}-k_{3} x_{3}
\end{aligned}
$$

Next, we consider the quadratic Lyapunov function

$$
V\left(x_{1}, x_{2}, x_{3}, e_{a}, e_{b}, e_{c}, e_{d}\right)=\frac{1}{2}\left(x_{1}^{2}+x_{2}^{2}+x_{3}^{2}+e_{a}^{2}+e_{b}^{2}+e_{c}^{2}+e_{d}^{2}\right)
$$

which is a positive definite function on $R^{7}$.

Note also that

$$
\dot{e}_{a}=-\dot{\hat{a}}, \quad \dot{e}_{b}=-\dot{\hat{b}}, \quad \dot{e}_{c}=-\dot{\hat{c}}, \quad \dot{e}_{d}=-\dot{\hat{d}}
$$

Differentiating $V$ along the trajectories of (7) and using (9), we obtain

$$
\begin{aligned}
\dot{V}= & -k_{1} x_{1}^{2}-k_{2} x_{2}^{2}-k_{3} x_{3}^{2}+e_{a}\left[x_{1}\left(x_{2}-x_{1}\right)-\dot{\hat{a}}\right]+e_{b}\left(x_{1} x_{2}-\dot{\hat{b}}\right) \\
& +e_{c}\left(x_{2}^{2}-\dot{\hat{c}}\right)+e_{d}\left(-x_{3}^{2}-\dot{\hat{d}}\right)
\end{aligned}
$$

In view of Eq. (10), the estimated parameters are updated by the following law:

$$
\begin{aligned}
& \dot{\hat{a}}=x_{1}\left(x_{2}-x_{1}\right)+k_{4} e_{a} \\
& \dot{\hat{b}}=x_{1} x_{2}+k_{5} e_{b} \\
& \dot{\hat{c}}=x_{2}^{2}+k_{6} e_{c} \\
& \dot{\hat{d}}=-x_{3}^{2}+k_{7} e_{d}
\end{aligned}
$$

where $k_{4}, k_{5}, k_{6}$ and $k_{7}$ are positive constants.

Next, we prove the following result. 
Theorem1. The controlled Cai system (1) with unknown parameters is globally and exponentially stabilized for all initial conditions $x(0) \in R^{3}$ by the adaptive control law (4), where the update law for the parameters is given by (11) and $k_{i},(i=1, \ldots, 7)$ are positive constants.

Proof. Substituting (11) into (10), we get

$$
\dot{V}=-k_{1} x_{1}^{2}-k_{2} x_{2}^{2}-k_{3} x_{3}^{2}-k_{4} e_{a}^{2}-k_{5} e_{b}^{2}-k_{6} e_{c}^{2}-k_{7} e_{d}^{2}
$$

which is a negative definite function on $R^{7}$.

Thus, by Lyapunov stability theory [30], it is immediate that the controlled Cai system (7) is globally exponentially stable and also that the parameter estimation errors $e_{a}, e_{b}, e_{c}, e_{d}$ exponentially converge to zero with time.

This completes the proof.

\subsection{Numerical Results}

For the numerical simulations, the fourth order Runge-Kutta method is used to solve the chaotic system (3) with the adaptive control law (4) and the parameter update law (11).

The parameters of the Cai system (3) are selected as

$$
a=20, \quad b=14, \quad c=10.6 \text { and } d=2.8
$$

For the adaptive and update laws, we take

$$
k_{i}=5, \quad(i=1,2, \ldots, 7) \text {. }
$$

Suppose that the initial values of the estimated parameters are

$$
\hat{a}(0)=9, \hat{b}(0)=22, \quad \hat{c}(0)=14, \hat{d}(0)=5
$$

The initial state of the controlled Cai system (3) is taken as

$$
x_{1}(0)=9, \quad x_{2}(0)=24, \quad x_{3}(0)=-20
$$

When the adaptive control law (4) and the parameter update law (11) are used, the controlled modified Cai system converges to the equilibrium $E_{0}=(0,0,0)$ exponentially as shown in Figure 2 .

The time-history of the parameter estimates is shown in Figure 3.

The time-history of the parameter estimation errors is shown in Figure 4. 
International Journal on Cybernetics \& Informatics ( IJCI) Vol.1, No.3, June 2012

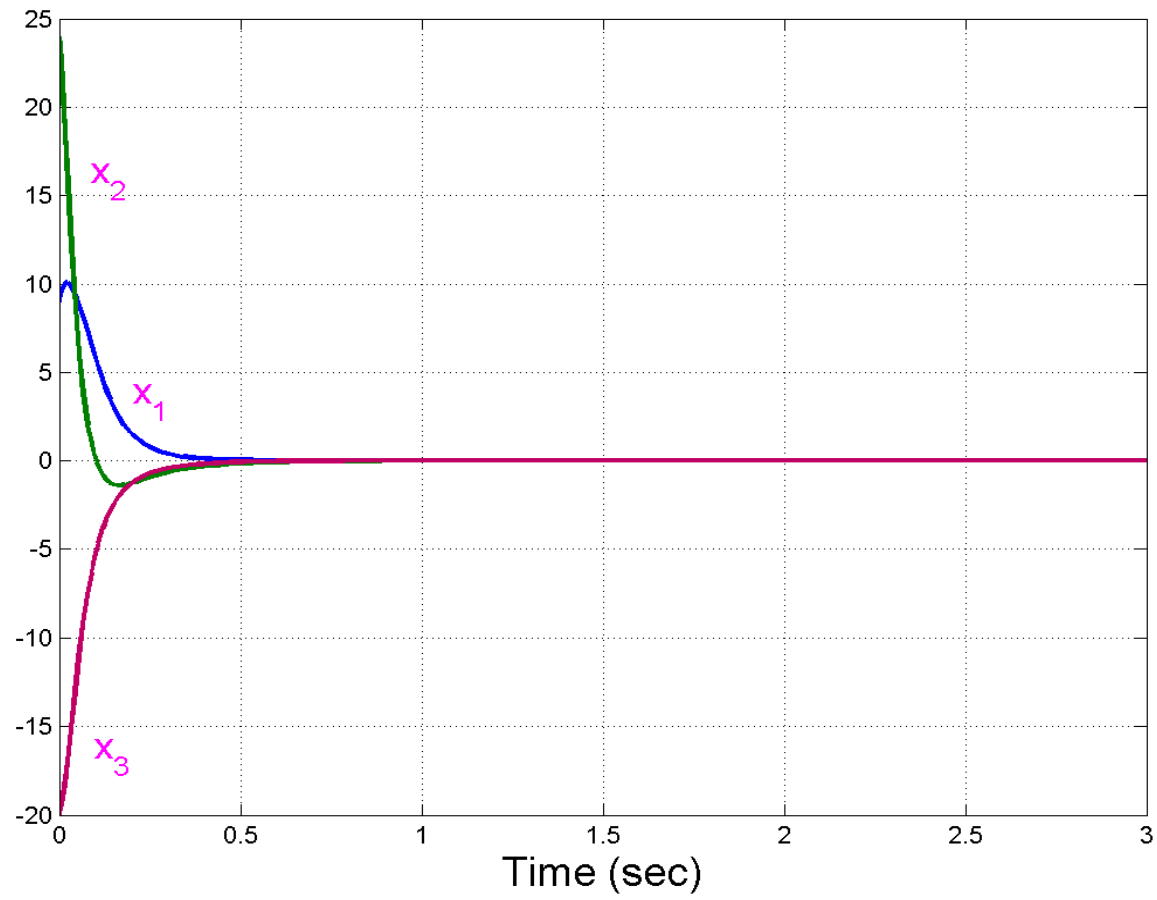

Figure 2. Time Responses of the Controlled Cai System

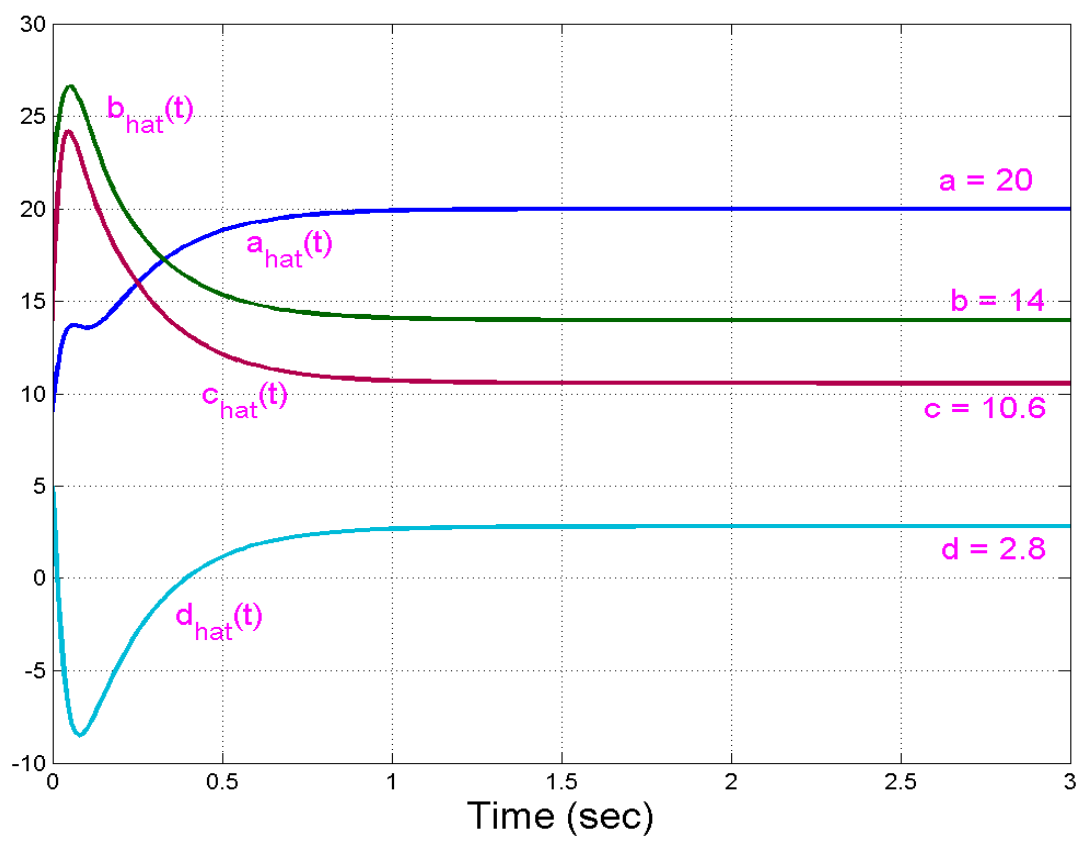

Figure 3. Time-History of the Parameter Estimates $\hat{a}(t), \hat{b}(t), \hat{c}(t), \hat{d}(t)$ 
International Journal on Cybernetics \& Informatics ( IJCI) Vol.1, No.3, June 2012

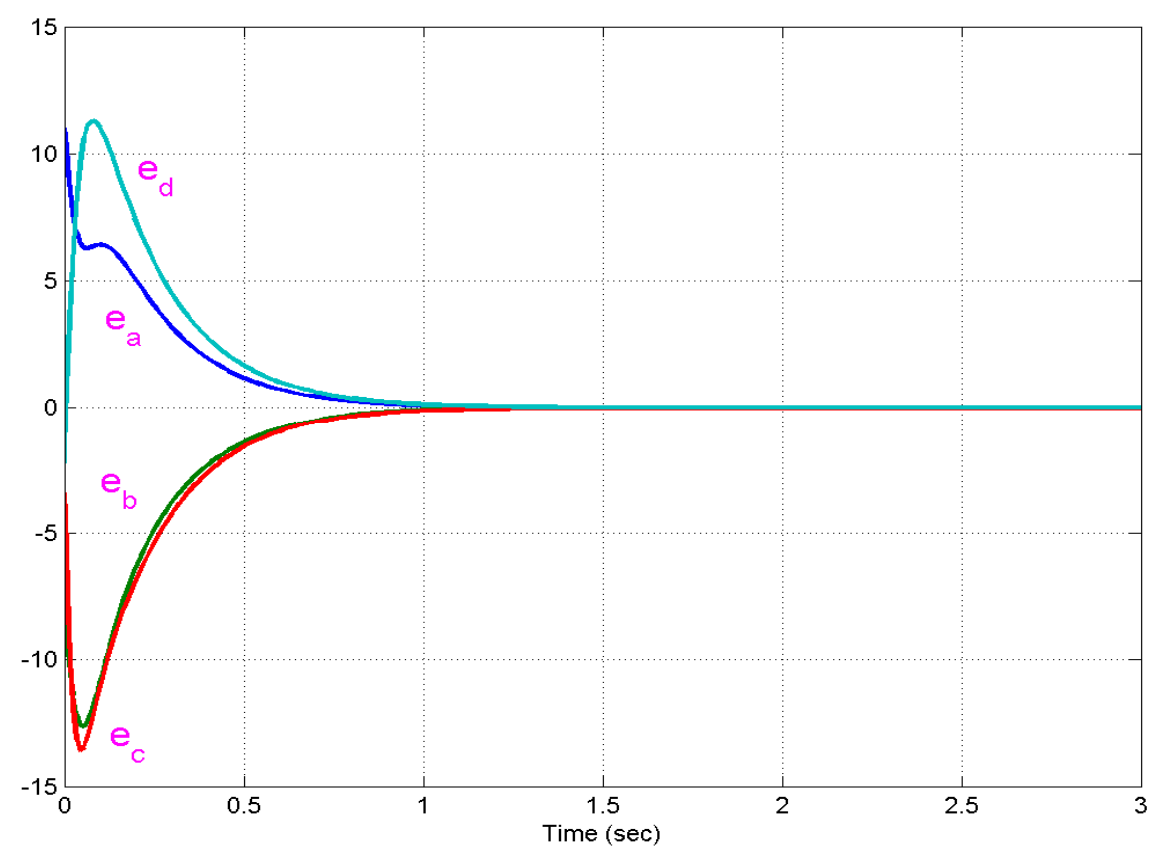

Figure 4. Time-History of the Parameter Estimates $e_{a}, e_{b}, e_{c}, e_{d}$

\section{Adaptive Synchronization of Identical Cai ChaOtic Systems}

\subsection{Theoretical Results}

In this section, we discuss the adaptive synchronization of identical Cai systems (2007) with unknown parameters.

As the master system, we consider the Cai dynamics described by

$$
\begin{aligned}
& \dot{x}_{1}=a\left(x_{2}-x_{1}\right) \\
& \dot{x}_{2}=b x_{1}+c x_{2}-x_{1} x_{3} \\
& \dot{x}_{3}=x_{1}^{2}-d x_{3}
\end{aligned}
$$

where $x_{i},(i=1,2,3)$ are the state variables and $a, b, c, d$ are unknown system parameters.

As the slave system, we consider the controlled Cai system described by

$$
\begin{aligned}
& \dot{y}_{1}=a\left(y_{2}-y_{1}\right)+u_{1} \\
& \dot{y}_{2}=b y_{1}+c y_{2}-y_{1} y_{3}+u_{2} \\
& \dot{y}_{3}=y_{1}^{2}-d y_{3}+u_{3}
\end{aligned}
$$


where $y_{i},(i=1,2,3)$ are the state variables and $u_{i},(i=1,2,3)$ are adaptive controllers to be designed.

The synchronization error is defined by

$$
\begin{aligned}
& e_{1}=y_{1}-x_{1} \\
& e_{2}=y_{2}-x_{2} \\
& e_{3}=y_{3}-x_{3}
\end{aligned}
$$

Then the error dynamics is obtained as

$$
\begin{aligned}
& \dot{e}_{1}=a\left(e_{2}-e_{1}\right)+u_{1} \\
& \dot{e}_{2}=b e_{1}+c e_{2}-y_{1} y_{3}+x_{1} x_{3}+u_{2} \\
& \dot{e}_{3}=-d e_{3}+y_{1}^{2}-x_{1}^{2}+u_{3}
\end{aligned}
$$

Let us now define the adaptive control functions $u_{1}(t), u_{2}(t), u_{3}(t)$ as

$$
\begin{aligned}
& u_{1}=-\hat{a}\left(e_{2}-e_{1}\right)-k_{1} e_{1} \\
& u_{2}=-\hat{b} e_{1}-\hat{c} e_{2}+y_{1} y_{3}-x_{1} x_{3}-k_{2} e_{2} \\
& u_{3}=\hat{d} e_{3}-y_{1}^{2}+x_{1}^{2}-k_{3} e_{3}
\end{aligned}
$$

where $\hat{a}, \hat{b}, \hat{c}$ and $\hat{d}$ are estimates of the parameters $a, b, c$ and $d, \quad$ respectively, and $k_{i},(i=1,2,3)$ are positive constants.

Substituting the control law (17) into (16), we obtain the error dynamics as

$$
\begin{aligned}
& \dot{e}_{1}=(a-\hat{a})\left(e_{2}-e_{1}\right)-k_{1} e_{1} \\
& \dot{e}_{2}=(b-\hat{b}) e_{1}+(c-\hat{c}) e_{2}-k_{2} e_{2} \\
& \dot{e}_{3}=-(d-\hat{d}) e_{3}-k_{3} e_{3}
\end{aligned}
$$

Let us now define the parameter errors as

$$
e_{a}=a-\hat{a}, e_{b}=b-\hat{b}, e_{c}=c-\hat{c}, e_{d}=d-\hat{d}
$$

Substituting (19) into (18), the error dynamics simplifies to

$$
\begin{aligned}
& \dot{e}_{1}=e_{a}\left(e_{2}-e_{1}\right)-k_{1} e_{1} \\
& \dot{e}_{2}=e_{b} e_{1}+e_{c} e_{2}-k_{2} e_{2} \\
& \dot{e}_{3}=-e_{d} e_{3}-k_{3} e_{3}
\end{aligned}
$$

Consider the quadratic Lyapunov function 


$$
V\left(e_{1}, e_{2}, e_{3}, e_{a}, e_{b}, e_{c}, e_{d}\right)=\frac{1}{2}\left(e_{1}^{2}+e_{2}^{2}+e_{3}^{2}+e_{a}^{2}+e_{b}^{2}+e_{c}^{2}+e_{d}^{2}\right)
$$

which is a positive definite function on $R^{7}$.

Note also that

$$
\dot{e}_{a}=-\dot{\hat{a}}, \quad \dot{e}_{b}=-\dot{\hat{b}}, \quad \dot{e}_{c}=-\dot{\hat{c}}, \quad \dot{e}_{d}=-\dot{\hat{d}}
$$

Differentiating $V$ along the trajectories of (20) and using (22), we obtain

$$
\begin{aligned}
\dot{V}= & -k_{1} e_{1}^{2}-k_{2} e_{2}^{2}-k_{3} e_{3}^{2}+e_{a}\left[e_{1}\left(e_{2}-e_{1}\right)-\dot{\hat{a}}\right]+e_{b}\left(e_{1} e_{2}-\dot{\hat{b}}\right) \\
& +e_{c}\left(e_{2}^{2}-\dot{\hat{c}}\right)+e_{d}\left(-e_{3}^{2}-\dot{\hat{d}}\right)
\end{aligned}
$$

In view of Eq. (23), the estimated parameters are updated by the following law:

$$
\begin{aligned}
& \dot{\hat{a}}=e_{1}\left(e_{2}-e_{1}\right)+k_{4} e_{a} \\
& \dot{\hat{b}}=e_{1} e_{2}+k_{5} e_{b} \\
& \dot{\hat{c}}=e_{2}^{2}+k_{6} e_{c} \\
& \dot{\hat{d}}=-e_{3}^{2}+k_{7} e_{d}
\end{aligned}
$$

where $k_{4}, k_{5}, k_{6}$ and $k_{7}$ are positive constants.

Theorem 2. The identical Cai systems (13) and (14) with unknown parameters are globally and exponentially synchronized for all initial conditions by the adaptive control law (17), where the update law for parameters is given by $(24)$ and $k_{i},(i=1, \ldots, 7)$ are positive constants.

Proof. Substituting (24) into (23), we get

$$
\dot{V}=-k_{1} e_{1}^{2}-k_{2} e_{2}^{2}-k_{3} e_{3}^{2}-k_{4} e_{a}^{2}-k_{5} e_{b}^{2}-k_{6} e_{c}^{2}-k_{7} e_{d}^{2}
$$

From (25), we find that $\dot{V}$ is a negative definite function on $R^{6}$.

Thus, by Lyapunov stability theory [30], it is immediate that the synchronization error and the parameter error decay to zero exponentially with time for all initial conditions.

\subsection{Numerical Results}

For the numerical simulations, the fourth order Runge-Kutta method is used to solve the two systems of differential equations (13) and (14) with the adaptive control law (17) and the parameter update law (24).

We take the parameter values as in the chaotic case, viz. 


$$
a=20, b=14, c=10.6, d=2.8
$$

We take the positive constants $k_{i},(i=1, \ldots, 7)$ as

$$
k_{i}=5 \text { for } i=1,2, \ldots, 7 \text {. }
$$

Suppose that the initial values of the estimated parameters are

$$
\hat{a}(0)=16, \hat{b}(0)=4, \hat{c}(0)=8, \quad \hat{d}(0)=5
$$

We take the initial values of the master system (13) as

$$
x_{1}(0)=-5, \quad x_{2}(0)=25, \quad x_{3}(0)=14
$$

We take the initial values of the slave system (14) as

$$
y_{1}(0)=24, \quad y_{2}(0)=10, \quad y_{3}(0)=-9
$$

Figure 5 shows the adaptive chaos synchronization of the identical Cai systems.

Figure 6 shows the time-history of the synchronization error $e_{1}, e_{2}, e_{3}$.

Figure 7 shows the time-history of the parameter estimates $\hat{a}(t), \hat{b}(t), \hat{c}(t), \hat{d}(t)$.

Figure 8 shows the time-history of the parameter estimation errors $e_{a}, e_{b}, e_{c}, e_{d}$.


Figure 5. Adaptive Synchronization of the Cai Systems 
International Journal on Cybernetics \& Informatics ( IJCI) Vol.1, No.3, June 2012

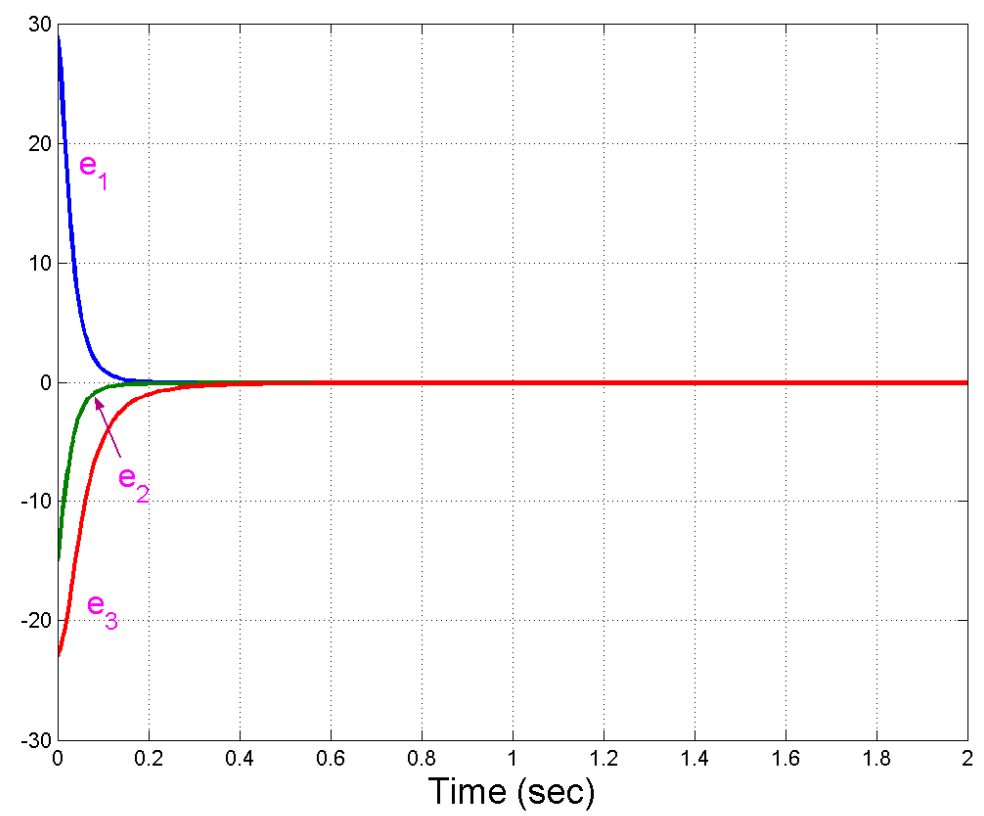

Figure 6. Time-History of the Synchronization Error $e_{1}, e_{2}, e_{3}$

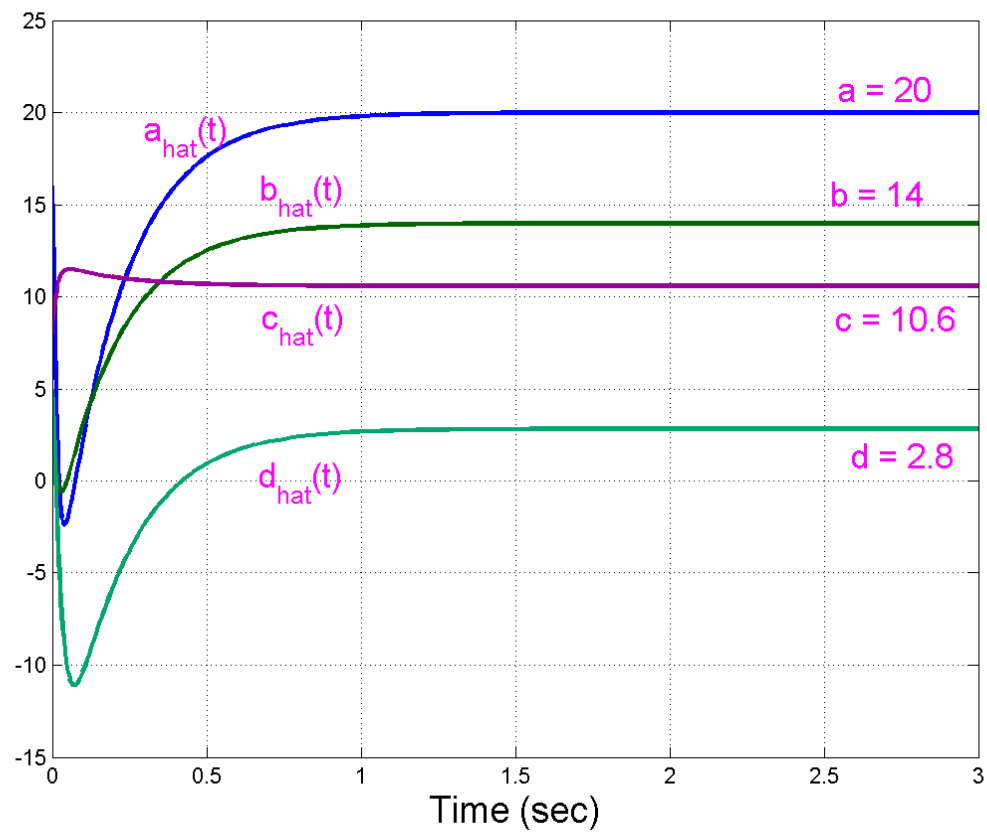

Figure 7. Time-History of the Parameter Estimates $\hat{a}(t), \hat{b}(t), \hat{c}(t)$ 


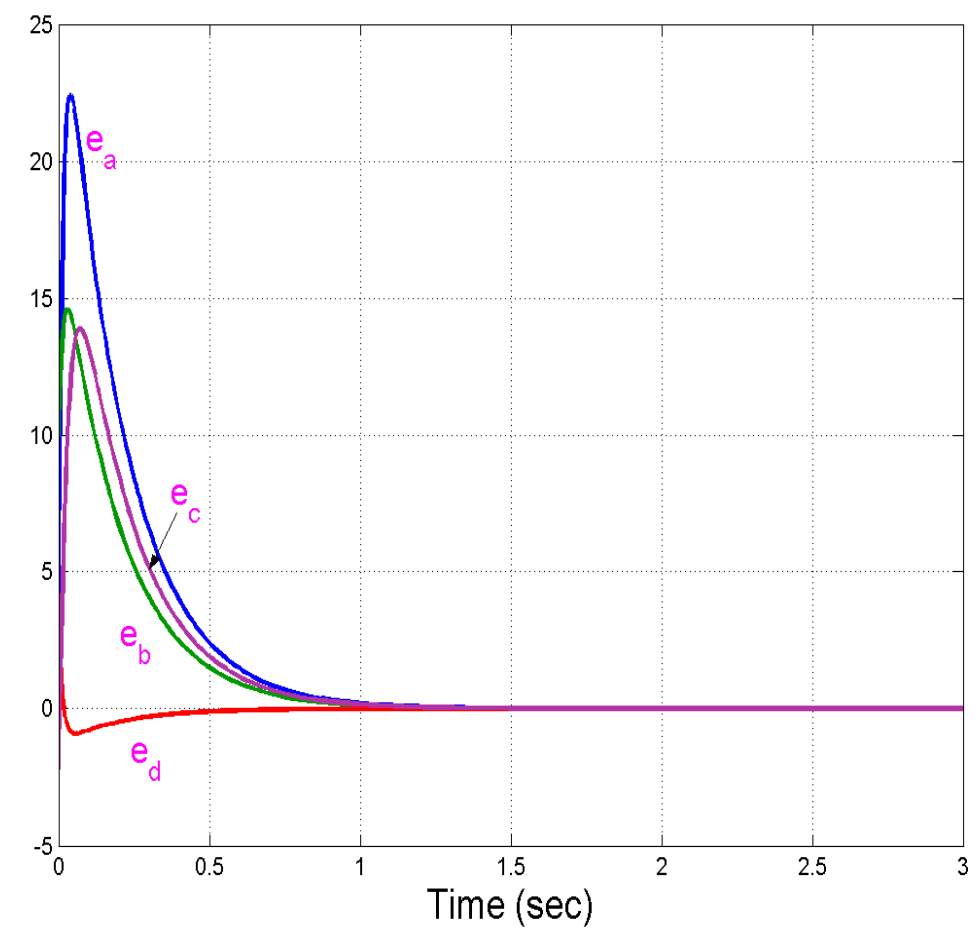

Figure 8. Time-History of the Parameter Estimation Error $e_{a}, e_{b}, e_{c}$

\section{Conclusions}

In this paper, we derived new results for the design of adaptive control and synchronization of the Cai system (2007) with unknown system parameters. First, we designed adaptive control laws to stabilize the Cai system to its unstable equilibrium point at the origin based on the adaptive control theory and Lyapunov stability theory. Then we derived adaptive synchronization scheme and update law for the estimation of system parameters for the identical Cai systems with unknown parameters. Our synchronization schemes were established using Lyapunov stability theory. Since the Lyapunov exponents are not required for these calculations, the proposed adaptive control method is very effective and convenient to achieve chaos control and synchronization of the Cai system. Numerical simulations are shown to depict the effectiveness of the proposed adaptive control and synchronization schemes.

\section{REFERENCES}

[1] Lorenz, E.N. (1963) "Deterministic nonperiodic flow," J. Atmos. Phys. Vol. 20, pp 131-141.

[2] Rössler, O.E. (1976) “An equation for continuous chaos," Physics Letters A, Vol. 57, pp 397-398.

[3] Wang, X., Tian, L. \& Yu, L. (2006) "Adaptive control and slow manifold analysis of a new chaotic system,” Internat. J. Nonlinear Science, Vol. 21, pp 43-49.

[4] Sun, M., Tian, L., Jiang, S. \& Xun, J. (2007) "Feedback control and adaptive control of the energy resource chaotic system," Chaos, Solitons \& Fractals, Vol. 32, pp 168-180.

[5] Pecora, L.M. \& Carroll, T.L. (1990) "Synchronization in chaotic systems”, Phys. Rev. Lett., Vol. 64, pp 821-824. 
International Journal on Cybernetics \& Informatics ( IJCI) Vol.1, No.3, June 2012

[6] Lakshmanan, M. \& Murali, K. (1996) Nonlinear Oscillators: Controlling and Synchronization, World Scientific, Singapore.

[7] Han, S.K., Kerrer, C. \& Kuramoto, Y. (1995) "Dephasing and bursting in coupled neural oscillators", Phys. Rev. Lett., Vol. 75, pp 3190-3193.

[8] Blasius, B., Huppert, A. \& Stone, L. (1999) "Complex dynamics and phase synchronization in spatially extended ecological system”, Nature, Vol. 399, pp 354-359.

[9] Feki, M. (2003) "An adaptive chaos synchronization scheme applied to secure communication", Chaos, Solitons and Fractals, Vol. 18, pp 141-148.

[10] Murali, K. \& Lakshmanan, M. (1998) "Secure communication using a compound signal from generalized synchronizable chaotic systems", Phys. Rev. Lett. A, Vol. 241, pp 303-310.

[11] Yang, T. \& Chua, L.O. (1999) "Control of chaos using sampled-data feedback control”, Internat. J. Bifurcat. Chaos, Vol. 9, pp 215-219.

[12] Ott, E., Grebogi, C. \& Yorke, J.A. (1990) "Controlling chaos", Phys. Rev. Lett., Vol. 64, pp 11961199.

[13] Park, J.H. \& Kwon, O.M. (2003) “A novel criterion for delayed feedback control of time-delay chaotic systems", Chaos, Solitons and Fractals, Vol. 17, pp 709-716.

[14] Yu, Y.G. \& Zhang, S.C. (2006) "Adaptive backstepping synchronization of uncertain chaotic systems", Chaos, Solitons and Fractals, Vol. 27, pp 1369-1375.

[15] Ho, M.C. \& Hung, Y.C. (2002) "Synchronization of two different chaotic systems by using generalized active control", Physics Letters A, Vol. 301, pp 424-428.

[16] Huang, L., Feng, R. \& Wang, M. (2004) "Synchronization of chaotic systems via nonlinear control", Physics Letters A, Vol. 320, pp 271-275.

[17] Tang, R.A., Liu, Y.L. \& Xue, J.K. (2009) "An extended active control for chaos synchronization," Physics Letters A, Vol. 373, No. 6, pp 1449-1454.

[18] Sundarapandian, V. (2011) "Output regulation of the Sprott-G chaotic system by state feedback control”, International Journal of Instrumentation and Control Systems, Vol. 1, No. 1, pp 20-30.

[19] Sundarapandian, V. (2011) "Global chaos synchronization of Lorenz and Pehlivan chaotic systems by nonlinear control", International Journal of Advances in Science and Technology, Vol. 2, No. 3, pp 19-28.

[20] Lei, Y., Xu, W. \& Zheng, H. (2005) "Synchronization of two chaotic nonlinear gyros using active control," Physics Letters A, Vol. 343, No. 1, pp 153-158.

[21] Liao, T.L. \& Tsai, S.H. (2000) "Adaptive synchronization of chaotic systems and its applications to secure communications", Chaos, Solitons and Fractals, Vol. 11, pp 1387-1396.

[22] Park, J.H., Lee, S.M. \& Kwon, O.M. (2007) "Adaptive synchronization of Genesio-Tesi chaotic system via a novel feedback control,” Physics Letters A, Vol. 371, pp 263-270.

[23] Sundarapandian, V. (2011) "Adaptive control and synchronization of hyperchaotic Chen system", International Journal of Information Technology Convergence and Services, Vol. 1, No. 3, pp 22-33.

[24] Sundarapandian, V. (2011) "Adaptive synchronization of hyperchaotic Lorenz and hyperchaotic Lü systems", International Journal of Instrumentation and Control Systems, Vol. 1, No. 1, pp 1-18.

[25] Sundarapandian, V. (2011) "Adaptive control and synchronization of hyperchaotic Cai system", International Journal of Control Theory and Computer Modeling, Vol. 1, No. 1, pp 1-13.

[26] Konishi, K., Hirai, M. \& Kokame, H. (1998) "Sliding mode control for a class of chaotic systems", Physics Letters A, Vol. 245, pp 511-517.

[27] Sundarapandian, V. (2011) "Sliding mode controller design for synchronization of Shimizu-Morioka chaotic system”, International Journal of Information Sciences and Techniques, Vol. 1, No. 1, pp 2029.

[28] Sundarapandian, V. (2011) "Global chaos synchronization of four-wing chaotic systems by sliding mode control”, International Journal of Control Theory and Computer Modeling, Vol., 1 No. 1, pp 15-31.

[29] Cai, G. \& Tan, Z. (2007) "Chaos synchronization of a new chaotic system via nonlinear control," Journal of Uncertain Systems, Vol. 1, No. 3, pp 235-240.

[30] Hahn, W. (1967) The Stability of Motion, Springer, New York. 


\section{Author}

Dr. V. Sundarapandian earned his Doctor of Science degree in Electrical and Systems Engineering from Washington University, St. Louis, USA in May 1996. He is a Professor at the R \& D Centre at Vel Tech Dr. RR \& Dr. SR Technical University, Chennai, Tamil Nadu, India. He has published over 260 papers in refereed international journals. He has published over 170 papers in National and International Conferences. $\mathrm{He}$ is the Editor-in-Chief of the AIRCC Journals - International Journal of Instrumentation and Control Systems, International Journal of Control Systems and Computer Modelling, and International Journal of Information Technology, Control and Automation. His research interests are Linear and Nonlinear Control Systems,

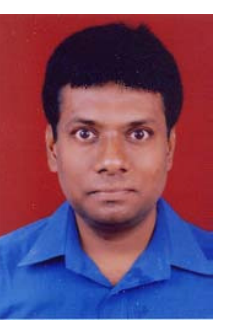
Chaos Theory and Control, Soft Computing, Optimal Control, Operations Research, Mathematical Modelling and Scientific Computing. He has delivered several Key Note Lectures on Control Systems, Chaos Theory and Control, Scientific Computing using MATLAB and SCILAB 